\title{
LA COMPRENSIÓN INFANTIL DE LA EMOCIÓN EN EL SISTEMA DE GUIDADO
}

\author{
GHILDREN'S UNDERSTANDING OF \\ EMOTION IN THE SYSTEM OF GARE
}

CRISTINA SANZ GUERRA ${ }^{1}$ Y PURIFICACIÓN SIERRA GARCÍA ${ }^{2}$

Cómo referenciar este artículo/How to reference this article:

Sanz Guerra, C. y Sierra García, P. (2017). La comprensión infantil de la emoción en el sistema de cuidado [Children's Understanding of Emotion in the System of Care]. Acción Psicológica, 14(2), 241-252. https://doi.org/10.5944/ap.14.2.19365

\section{Resumen}

La relación madre-hijo se ha estudiado tradicionalmente desde la perspectiva de la teoría del apego. Pero, además, es un fenómeno psicológico y social al que el niño dota de sentido y que trata de comprender. Para explorar la comprensión infantil de esta dimensión de la relación, hemos entrevistado a 85 niños de entre siete y nueve años mediante una entrevista semiestructurada con cuatro historias, en las que se relacionaban el Sistema de Apego y el Sistema de Cuidado. El análisis de las respuestas de los niños muestra que el $97 \%$ atribuyen malestar en la madre en relación con la vivencia de amenaza del hijo. Los niños de estas edades poseen ya una comprensión del Sistema de Cuidado de la madre en
\end{abstract}

conexión con el Sistema de Apego del hijo. La responsabilidad y la empatía de la madre forman parte de las respuestas de los niños de estas edades como activadores de su comportamiento de cuidado y protección hacia el hijo. Las representaciones de los niños pueden organizarse en niveles de comprensión cada vez más sofisticados y complejos. Conocer los sentimientos y motivaciones que los niños atribuyen a la madre en su rol de cuidadora principal y la visión infantil de las leyes emocionales que rigen las relaciones afectivas madre-hijo, puede ofrecernos nuevas perspectivas acerca de cómo se construyen las relaciones de apego y resultar útil en la detección e intervención en apegos no saludables, en ambos miembros de la díada.

Palabras clave: Sistema de cuidado; Sistema de apego; Compresión infantil; Modelo interno de trabajo.

Correspondencia: Purificación Sierra García. Facultade de Psicoloxía, Universidad Nacional de Educación a Distancia (España). Email: psierra@psi.uned.es

${ }^{1}$ Conserjería de Educación, Junta de Extremadura, España.

${ }^{2}$ Universidad Nacional de Educación a Distancia (UNED), España.

Recibido: 7 agosto de 2017.

Aceptado: 16 de noviembre de 2017. 


\begin{abstract}
The mother-child relationship has traditionally been studied from the perspective of attachment theory. But in addition, they are a psychological and social phenomenon to which the child gives meaning and tries to understand. To explore the child's understanding of this dimension of the relationship, we interviewed 85 children between 7 and 9 years old through a semistructured interview with four stories, in which the Attachment System and the System of Care were related. The analysis of the children's responses shows that $97 \%$ attribute discomfort in the mother in relation to the experience of threat of the child. Children of these ages already have an understanding of the Mother's System of Care in connection with the child's attachment system. The responsibility and empathy of the mother are part of the responses of children of these ages as activators of their behavior of care and protection for the child. Children's representations can be organized at increasingly sophisticated and complex levels of understanding. Knowing the feelings and motivations that children attribute to the mother in her role of primary caregiver and the child's vision of the emotional laws that govern affective mother-child relationships, can offer us new perspectives on how attachment relationships are built and be useful in the detection and intervention in unhealthy attachments, in both members of the dyad.
\end{abstract}

Keywords: System of care; Attachment system; Child's understanding; Internal working model.

\section{Introducción}

La relación afectiva entre el niño y su cuidador es una construcción diádica, en la que ambos miembros aportan objetivos, cogniciones y emociones propias. Desde que en 1969 Bowlby propusiera tu teoría del apego, ésta se ha convertido en el paradigma hegemónico en el estudio de las relaciones madre-hijo.

La relación afectiva entre el niño y su cuidador es una construcción diádica, en la que ambos miembros aportan objetivos, cogniciones y emociones propias. La dinámica de la vinculación de apego supone la puesta en marcha de comportamientos por parte del niño con el objetivo de buscar y mantener la proximidad de la madre y están organizados en el Sistema de Apero (SA). El aprendizaje y el desarrollo de herramientas cognitivas durante la primera infancia la interiorización de la disponibilidad (o no) de la figura de apego en un Modelo Interno de Trabajo (MIT) (Bowlby, 1969/1982). El MIT contiene información de sí mismo, de la madre y de la relación. El niño va conformando una imagen cada vez más compleja de la madre como ser psicológico, como un individuo independiente con objetivos y emociones propias gracias a la experiencia y el desarrollo cognitivo. Esto permite que la relación sea cada vez más completa y flexible (Bowlby, 1988). La representación mental es el componente central en la explicación del comportamiento socioemocional y el ajuste psicológico e interpersonal más allá de la infancia (Mikuliner y Shaver, 2005). Aunque el MIT incluye emociones de ambos miembros de la díada, Bowlby no contempla las emociones de la madre en relación con las tareas específicas de protección y cuidado.

Complementario a la representación infantil de la relación, Solomon y George $(1986,2008)$ proponen un Sistema de Representación de Cuidado o Sistema de Cuidado (SC) en la madre. Esta representación contiene cogniciones y emociones sobre las competencias y estrategias para cubrir las necesidades del hijo y la eficacia de las mismas. La evaluación de dicha eficacia, repercutiría en sus prácticas de crianza (George y Solomon, 2008).

La Teoría de la Conexión (Bell, 2010, 2012; Bell y Richard, 2000), ahonda en la cualidad de las emociones que activan el SC. Éstas serían la empatía (intención emocional y cognitiva de conocer y entender las necesidades del niño) y la responsabilidad (intención emocional y cognitiva de ayudar y proteger al niño). Ambos componentes subyacen a la eficacia del cuidado y las prácticas de crianza y, por tanto, a la cualidad de la relación afectiva. Ambas propuestas sobre el SC carecen por el momento de desarrollo empírico.

Los estudios sobre la representación infantil de la relación madre-hijo, se han realizado exclusivamente desde 
una perspectiva evaluativa del apego mediante estrategias de completamiento de historias (e.g., Walsh, Symons y McGrath, 2004). Su dinámica consiste en analizar el discurso infantil que será el reflejo de la historia afectiva de la díada $\mathrm{y}$, desde él, establecer patrones de apego. El niño verbalizará sus expectativas de la respuesta de la madre ante situaciones activadoras del SA, aquellas en las que el niño se siente amenazado.

Dada la potencia y repercusión de la teoría del apego, pareciera que este enfoque agota las posibilidades del estudio infantil de la relación madre-hijo. Pero éstas pueden ser abordadas desde otros planteamientos igualmente interesantes para nuestro avance en el conocimiento y comprensión e intervención en el desarrollo infantil y el apego.

En la relación madre-hijo confluyen distintas dimensiones. Por una parte, se trata de relaciones afectivas de naturaleza específica con repercusiones fundamentales en el desarrollo y la salud mental, como señala la teoría del apego (Bowlby, 1969/1982). Desde esta perspectiva, es un proceso psicobiológico que no requiere de comprensión en la infancia (mientras emerge y se consolida). Pero desde la psicología del desarrollo, las relaciones afectivas madre-hijo se encuentran dentro de la categoría de fenómenos sociales y psicológicos porque: (a) son procesos que se producen en el contexto social de las relaciones entre las personas (Kalish y Lawson, 2008), (b) ambos participantes desempeñan dentro de ella un rol (diádico) que se circunscribe a la relación, y (c) en ella confluyen las dimensiones cognitiva y emocional de ambos miembros.

Comprender el mundo que le rodea es una de las características intrínseca a los seres humanos y fundamental en el desarrollo porque permite una actuación mucho más eficaz sobre dicha realidad (Delval, 2007). El niño el niño trata de dotar de sentido a todos los fenómenos sean del mundo físico, psicológico o social. Para ello forma representaciones o modelos que incluyen expectativas sobre los comportamientos de los participantes y las leyes que rigen dichos comportamientos. Estas representaciones se construyen en la interacción entre las competencias cognitivas y la experiencia social de los niños. Por ello, no son una copia de lo que el adulto le transmite sobre la realidad, sino que constituyen auténticas elaboraciones individuales que van evolucionando a lo largo de la infancia, desde atribuciones sencillas, basadas en aspectos concretos de la realidad, externos y desconectados, hasta otros más elaborados, inferenciales y abstractos, en los que los elementos se conectan de manera progresivamente más compleja y relacional (Delval, 2000; Kalish y Lawson, 2008).

La dificultad del estudio de la comprensión infantil de los fenómenos requiere indagar no sólo en la información que posee el niño sobre ellos, sino en su visión personal acerca de los elementos que los conformas y las relaciones que establece entre ellos, en esencia, qué significado les otorga. Para ello, es necesaria una estrategia que permita profundizar en las ideas íntimas de los niños. El método clínico-crítico de Piaget (1926) nos brinda esta posibilidad ya que supone indagar, en la comprensión infantil de los fenómenos a estudiar siguiendo el curso del pensamiento del niño, analizando la cualidad de los argumentos.

No contamos con estudios sobre la comprensión infantil de las relaciones afectivas madre-hijo. El objetivo general de nuestro trabajo, ha sido explorar la comprensión infantil de la dimensión psicológica de estas relaciones. En concreto, nos preguntábamos si el niño: (a) contemplaba la emoción como un activador del SC; (b) qué emoción subyacía esta activación y su justificación; (c) si poseía una representación diádica del SA y del SC y, por último, cómo se organizaba dicha representación.

Conocer los sentimientos y motivaciones que los niños atribuyen a la madre respecto a su rol de cuidadora principal y la visión infantil de las leyes emocionales que rigen las relaciones afectivas madre-hijo, puede ofrecernos nuevas perspectivas sobre cómo interpreta el niño el rol de protección de la madre, los elementos en torno a los cuáles se organiza la vivencia de la madre y su comportamiento como cuidadora principal. Ello no solamente nos conducirá a una mejor comprensión de cómo se construye las relaciones apego, sino que también puede resultar útil en la detección e intervención en ambos miembros de la díada en apegos no saludables. 


\section{Método}

\section{Participantes}

Dada la naturaleza del estudio y las exigencias de los centros, se envió una carta de presentación a la dirección y a las familias de los alumnos. Tras ello se impartieron charlas colectivas a los padres de los niños que se encontraban en los cursos $1^{\circ}, 2^{\circ}$ y $3^{\circ}$ de Educación Primaria (509 familias), cumplieran o no los requisitos de edad en ese momento puesto que la edad cronológica del alumnado varía a lo largo de los cursos en la previsión de que el estudio se haría con posterioridad a esta primera fase de petición de autorización. De ellas, no mostraron interés en el estudio 400 familias. En base a la ley de protección de datos, el colegio no aportó información de ninguna de ellas. El total de familias que aceptaron la participación en el estudio fueron 109, de las cuáles, en el momento de las entrevistas, sólo cumplían requisitos 85 , lo que constituyó el $\mathrm{N}$ total de la muestra.

Respecto a las características de la muestra, estuvo compuesta por 85 niños y niñas $(61 \%$ niñas y $39 \%$ niños), de edades comprendidas entre los siete y los nueve años, $M=8.26$ y $S D=0.61$ (rango 7.0-8.9). Todos los niños eran de nacionalidad españolas excepto uno de nacionalidad brasileña. Los niños asistían a tres centros públicos y un centro privado concertado en Mérida. La mayoría de los niños de la muestra (86\%) viven con ambos padres. Los padres de ocho niños estaban separados y convivían habitualmente con la madre. Tres niños procedían de familias monoparentales, siendo el progenitor la madre en dos casos y en uno, el padre. Respecto a la edad, los padres tuvieron una edad media $M=42.54$ $(S D=4.81)$, mientras que, en las madres, la edad media fue de 40,45 $(S D=4,52)$. Respecto al cuidador principal, el $60 \%$ (51) declaran que son ambos; el $20 \%(23.5)$ señalan a la madre como cuidador principal; $3.5 \%$ (3) contestan que es el padre quien se ocupa principalmente del niño/a y un $12.9 \%$ (11) no contestan.

\section{Procedimiento}

Previa autorización del equipo directivo de los centros educativos, se realizó una charla colectiva con los padres de los alumnos de entre siete y nueve años. Los niños cuyos padres firmaron un consentimiento informado aceptando participar en el estudio, fueron entrevistados dentro del horario escolar, de manera individual en el propio centro educativo, en un lugar destinado al efecto. Su duración aproximada fue de 15 minutos.

La entrevista fue presentada a los niños como una tarea desprovista de carácter evaluativo.

\section{Instrumentos}

Los padres completaron una ficha creada ad hoc en la que se incluían datos sobre la nacionalidad y aspectos de la conformación familiar.

De acuerdo con los objetivos de nuestro estudio se elaboró una entrevista semiestructurada: Entrevista para Niños sobre la Emoción y Responsabilidad en el Sistema de Cuidado (ENERSC).

Se compone de cuatro Historias en las que los protagonistas son una madre y su hijo. En la primera denominada Obligación Laboral, se narraba lo siguiente: Una madre está trabajando. Recibe una llamada del colegio de su hijo para decirle que se encuentra mal y debe ir a recogerlo para llevarle al médico. En la segunda Historia, denominada Descuido, una madre se encuentra en un centro comercial con su hijo. Hay mucha gente y lo lleva de la mano. De repente, se da cuenta de que ya no siente la mano de su hijo agarrándola, mira y no ve a su hijo, lo ha perdido. La tercera Historia expone que una madre va en coche a recoger a su hijo a clase de inglés. Entonces se pincha una rueda del coche. Esta Historia se denominó Imponderables. La cuarta y última Historia, Despiste, describe que una madre lleva a su hijo a un cumpleaños. Debe ir a recogerlo a las siete, pero se despista y cuando se da cuenta, ve que va a llegar tarde. Tras cada historia, se le preguntaba al niño: ¿Tú cómo crees que se siente la madre? y se le pedía que justificara su respuesta. En un segundo momento se le pedía al niño que señalara alguna solución para que el niño pudiera recibir los cuidados de 
la madre. Ante su respuesta, la entrevistadora señalaba que no era posible y se le volvía a preguntar sobre el sentimiento de la madre. A efectos de análisis, las respuestas de los niños a esta pregunta se codificaron como Escenario 2 (E2).

La entrevista se desarrollaba atendiendo al objetivo general de obtener información sobre la representación y comprensión de los niños, siguiendo el curso de su pensamiento, en términos Piagetianos, las creencias espontáneas de los niños (Piaget, 1926). En este estudio, presentaremos las respuestas relacionadas con los sentimientos que los niños atribuyen a la madre en ambos escenarios (E1) y (E2).

\section{Análisis de los datos}

El análisis de los datos se llevó a cabo en varias fases. En la primera, se codificaron las respuestas de los niños atendiendo a criterios sustantivos y conceptuales. Desde ellas, elaboramos categorías generales, atendiendo a los elementos de que constaban, su organización y relación entre los mismos. Estas categorías, dieron lugar a su agrupación en distintos Niveles de complejidad. El Nivel 1, al que hemos denominado Representación ligada a Rasgos Concretos de la Situación, contiene respuestas en las que los niños, aun reconociendo el malestar de la madre, se ciñen a los elementos descriptivos que aparecen en las Historias y que aporta el entrevistador, como en el caso de Leire $(8 ; 10)$ : "¿por qué se siente mal (la madre) ${ }^{5}$ ? Porque se le ha pinchado la rueda del coche" o Ángela (7; 3): "¿por qué se siente triste (la madre)? Porque su jefe no le deja ir a por su hija".

En el Nivel 2, denominado Representación del Compromiso de Cuidado, se agrupan respuestas en las que los niños, además de tener en cuenta los acontecimientos narrados en las Historias, tienen en cuenta cómo estas circunstancias condicionan la consecución del objetivo (procurar protección al hijo, que el hijo se sienta protegido), desde una perspectiva del compromiso o responsabi-

Se presentan extractos literales de las entrevistas. Las preguntas de la entrevistadora aparecen en letra redonda y la respuesta de los niños en cursiva. lidad poco elaborada, como nos muestra Adrián $(8 ; 2)$ : "¿Qué crees que siente la madre cuando le llaman del cole porque se ha puesto malo? Se siente mal y preocupada ¿y por qué? Porque no puede ir al hospital a cuidarlo, tendrá que esperar hasta que llegue a casa. O Yaiza $(8 ; 6)$ : "¿Qué crees que siente la madre cuando se pincha la rueda? Enfadada, porque no puede ir a recoger a su hija")

En el siguiente Nivel de complejidad (Nivel 3) los argumentos de los niños mostraron representaciones en las que los argumentos sobre el malestar de la madre se organizaban en torno a la toma de conciencia de la activación de los Sistemas de Apego (en el niño) y de Cuidado (en la madre) así como los impedimentos para poder alcanzar los objetivos. De esta manera, en este Nivel, los argumentos contenían nociones de responsabilidad y empatía cualitativamente diferentes y mostrando una conexión emocional de la madre con el hijo. A este tercer Nivel se denominó Representación de una Conexión emocional. Algunos ejemplos de estas respuestas son las que aparecen a continuación: Paola $(8 ; 11)$ "¿Qué crees que siente la madre cuando se pincha la rueda? Pues está como así: "¡qué tengo que darme prisa! ¡qué mi hija a lo mejor se preocupa! jtengo que darme prisa!" "Está enfadada con ella misma por haber pinchado una rueda y triste porque tiene que ir a por su hija y no quiere dejarla más sola" o Miriam $(8 ; 10)$ que señala que la madre se siente "... triste porque piensa la hija que se ha olvidado y la ha dejado sola”.

Se contó con el apoyo de software de análisis cualitativo de datos (CAQDAS, Computer-Aided Qualitative Data Analysis), en concreto, el Atlas.ti 6.0 como herramienta principal para la categorización de las respuestas de los niños atendiendo a su contenido sustantivo. Un grupo de protocolos seleccionados al azar fueron codificados por un segundo juez. El porcentaje de acuerdo en el proceso de categorización fue del $90 \%$. Los desacuerdos en las categorizaciones se resolvieron mediante discusión.

Aunque el objetivo del trabajo es fundamentalmente cualitativo, atendiendo a la naturaleza de los datos se realizaron análisis estadísticos mediante Chi Cuadrado para analizar las diferencias entre los niveles. En una fase 
siguiente, estos datos han sido analizados de manera cuantitativa mediante el paquete estadístico SPSS 19.0.

\section{Resultados}

\section{Reconocimiento y valencia de la emoción de la madre}

De manera global, a la pregunta ¿qué crees que siente la madre (en cada una de las situaciones) ?, la mayoría de las respuestas de los niños (el $97 \%$ ) atribuyeron a la madre un sentimiento negativo o de malestar. La justificación de esta emoción se hallaba en que la madre no podía desplegar el SC (como en el caso de la historia () en el que no puede ir a buscarle al colegio cuando está enfermo porque está trabajando); o en que había no había sido eficaz en el mismo (como en el caso de la historia 4 que narra la pérdida del niño en el centro comercial). Esta emoción mayoritaria y su argumento será objeto de análisis en profundidad en el siguiente apartado.

Un $2 \%$ de las respuestas fueron "no sé" y un $1 \%$ fueron anecdóticas como que "también tiene su parte buena porque se puede quedar a trabajar, y asi luego cuando llegue si tarda mucho pues no le cae todo de golpe"). Aun siendo interesantes desde el punto de vista cualitativo, dado su carácter residual no se tomaron en consideración en las siguientes fases del análisis.

\section{Justificación de la emoción de la madre}

En relación con la representación infantil del motivo del malestar de la madre en las diferentes situaciones, como se ha dicho en el apartado correspondiente, encontramos tres tipos de respuestas que suponen Niveles crecientes de complejidad de las representaciones, tanto por los elementos que contienen como por la forma en la que se organizaban.

En la Tabla 1 se presenta la distribución de niños en los diferentes niveles y en ambos escenarios (E1) y (E2).

Como puede apreciarse, los argumentos de los niños de estas edades se agruparon, en mayor medida en el Nivel 2 en ambos Escenarios, siendo menor la diferencia entre el Nivel 2 y 3 en el Escenario 1 que entre el 2 y 3 en el Escenario 2.

Sólo un $20 \%$ de los niños se situaron en el Nivel 1. Representación ligada a Rasgos Concretos de la Situación. Las respuestas de los niños mostraron una representación en la que el SC y Apego se encuentran activados, pero por elementos concretos y sobresalientes de las Historias, los niños atribuyeron el malestar de la madre a los elementos descritos en ellas, tal y como señaló Lourdes $(7 ; 10)$ "se sentía triste porque su hija está mala". Estas argumentaciones basadas en elementos concretos de las historias, se hacen explícitas en referencia directa al SC (Aitana, 8;9, afirma que la madre se sentía "fastidiada, porque se le ha pinchado la rueda"). El porcentaje de niños que aportan argumentos de este nivel de concreción cuando se presenta el Escenario 2, es decir, cuando

Tabla 1

Determinantes de la emoción de la madre en los Escenario 1 y 2

\begin{tabular}{lll}
\hline Nivel de Representación & $\begin{array}{l}\text { Escenario 1 } \\
\text { (Historia inicial) } \\
\mathrm{N}(\%)\end{array}$ & $\begin{array}{l}\text { Escenario 2 } \\
\text { (Impedimento) } \\
\mathrm{N}(\%)\end{array}$ \\
\hline $\begin{array}{l}\text { Nivel 1 } \\
\text { Representación ligada a Rasgos Concretos de la Situación } \\
\quad \text { Nivel 2 }\end{array}$ & $\left.17(20 \%)^{*}\right)$ & $11(13 \%)$ \\
$\begin{array}{l}\text { Representación del Compromiso de Cuidado } \\
\quad \text { Nivel 3 }\end{array}$ & $38(45 \%)$ & $45(53 \%)$ \\
Representación de una Conexión emocional & $30(35 \%)$ & $29(34 \%)$ \\
\hline
\end{tabular}

Nota. ${ }^{(*)}$ Los niños pueden estar en diferentes niveles dependiendo de las historias. 
se introduce un impedimento ajeno a la voluntad de la madre por la que no puede alcanzar el objetivo del SC.es algo menor $(13 \%)$.

El mayor número de niños $(45 \%)$ se situó en el que hemos denominado Nivel 2 Representación del Compromiso de Cuidado. La característica de este Nivel es que se aportan organizaciones más complejas en sus explicaciones en torno al SC, situando el origen del malestar de la madre en la toma de conciencia (de la madre) de que no puede hacer frente a la demanda de protección del niño, por ejemplo, Sofía $(8 ; 5)$ : (la madre se sentía mal) "Porque... se le ha pinchado una rueda y ya no puede recoger a la niña." Las respuestas en las que los niños aluden al Sistema de Apego se refieren a que el malestar de la madre se origina en el potencial peligro que puede estar viviendo el hijo en el contexto de la historia y que, por tanto, requiere la activación de su SC para regular el sentimiento de amenaza en el niño. La madre puede sentir preocupación porque, como expresa Paula $(8 ; 11)$ “... a ver si la niña va a tener algo, que va a quedar enferma, que sea algo grave, ...".

Como puede observarse en la Tabla 1, las respuestas que se sitúan en este Nivel se incrementaron ligeramente ante el Escenario 2. Es decir, hay un mayor número de niños que argumentan esta emoción de la madre cuando se le presenta un nuevo impedimento para poder desplegar el SC.

Por último, un porcentaje algo menor de niños (el $35 \%$ ) dieron respuestas del Nivel 3., Representación de una Conexión emocional. Los argumentos que tomaban en consideración sobre el SC, giraban en torno a los sentimientos negativos que el niño atribuye a la madre (de

Consistencia en el Nivel de respuesta entre los Escenarios 1 y 2

\begin{tabular}{|c|c|c|c|c|c|}
\hline \multirow{2}{*}{\multicolumn{2}{|c|}{ Número de niños en cada Nivel y cada Escenario }} & \multicolumn{3}{|c|}{$\begin{array}{c}\text { Nivel de Respuesta } \\
\text { en el E2 } \\
\text { (Impedimento) }\end{array}$} & \multirow[t]{2}{*}{$\mathrm{N}$ total } \\
\hline & & Nivel 1 & Nivel 2 & Nivel 3 & \\
\hline Nivel de Respuesta & Nivel 1 & $29.4(5)$ & $47.1(8)$ & $23.5(4)$ & 17 \\
\hline E1. & Nivel 2 & $10.5(4)$ & $71.1(27)$ & $18.4(7)$ & 38 \\
\hline (Historia Inicial) & Nivel 3 & $6.7(2)$ & $33.3(10)$ & $60(1) 8$ & 30 \\
\hline $\mathrm{N}$ total & & $12.9(11)$ & $52.9(45)$ & $34.1(29)$ & 85 \\
\hline
\end{tabular}

malestar consigo misma) incluyendo sentimientos de culpa o vergüenza por haber faltado al Cuidado o el compromiso con el niño, en palabras de Paola $(8 ; 11)$ "Pues la madre se enfada con ella misma, porque debería haber estado atenta, o ponerse una alarma, debería haber estado atenta e ir a por su hija". Aquí también se incluyen sentimientos relacionados con la "obligación" de atender al hijo, o confusión o desorientación por tener que hacer frente a la disyuntiva de compaginar el Cuidado de su hijo con otras obligaciones, pero poniendo la obligación hacia el hijo por delante. Por ej. Daniel $(7 ; 6)$ : "Pues un dolor en la cabeza y, como si no supiera qué hacer, porque ¿tengo que ir a trabajar para ganar dinero o tengo que ir a por mi hijo a recogerle? Que no sabe, pero ella va a recoger a su hijo". De esta manera, los niños incluso son conscientes y se representan el dilema al que se encontrarían expuestas las madres. Por su parte, los argumentos relativos al $\mathrm{SA}$, se refirieron a la atribución de sentimientos negativos o de malestar de la madre causados por la emoción/vivencia que la madre prevé que tendrá el niño como consecuencia del acontecimiento narrado en la Historia. Paula $(8 ; 10)$ lo describe así: la madre se sentía "preocupada" porque "a su hija le da miedo quedarse sola".

No se han encontrado diferencias significativas en función del sexo entre los niveles de comprensión en ninguno de los Escenarios.

Pero ¿mantienen los niños la cualidad de sus razonamientos cuando se introduce un impedimento en la situación, de manera que la madre no pueda atender a las necesidades del hijo? Desde esta perspectiva, los resultados son los que se muestran en la Tabla 2.

\section{Tabla 2}


Como puede observarse, de los 17 de niños que aportaron respuestas propias del Nivel 1 cuando se les presentaba la Escena inicial (E1), 12 dieron respuestas más elaboradas cuando se introdujo el impedimento (E2). De ellos ocho aludieron ahora al sentimiento de malestar de la madre atendiendo a su Compromiso de Cuidado (Nivel 2) y 4 aportaron argumentos propios de un el Nivel de respuestas cualitativamente más complejo (Nivel 3: Conexión emocional).

Resulta interesante también que de los 38 niños que señalaron la emoción de malestar de la madre atendiendo al compromiso o responsabilidad en la protección del hijo en la primera Escena, cuando se les presentaba el segundo Escenario, la mayoría respondieron de manera consistente, situando sus respuestas en este mismo Nivel. Pocos niños dieron respuestas del Nivel anterior y algunos ya comenzaron a dar respuestas del Nivel superior ante la imposibilidad de la que la madre pudiera resolver la situación activando comportamientos de Cuidado.

Por su parte, de los 30 niños que aludieron a aspectos de empatía y compromiso de forma más clara y organizada, con sentimientos de un mayor Nivel abstracción y complejidad como se ha visto anteriormente, la mayoría (18) dieron respuestas acordes con este Nivel cuando se les señalaba que no podía solucioinarse la situación. Algo menos de la mitad "retrocedieron" a argumentos menos complejos y sólo alguno (dos) aportaron los argumentos más simples (aquellos relacionados con la propia situación).

Hemos encontrado diferencias significativas en el número de niños en los niveles que aportan diferentes argumentos en función de la introducción del impedimento en la Historia (Escenario 2) $\left(X^{2}=18.485\right.$ g.l. $=4$, $p=.001)$, Coeficiente de Contingencia $=423, p=.001$.

\section{Conclusiones}

El objetivo general de nuestro trabajo ha sido explorar la comprensión infantil de la relación madrehijo como fenómeno psicológico y social. Se trata de un campo de interés inexplorado y de especial utilidad para nuestro conocimiento de la comprensión infantil de la vinculación de apego, uno de los pilares del desarrollo socioemocial. Se han indagado estas representaciones mediante una Entrevista semiestructura creada ad hoc formada por Historias y preguntas encaminadas a hacer emerger el conocimiento y representación infantil de los elementos motivacionales y de activación del SC, concretamente, la emoción de la madre. La entrevista y su dinámica ponían en juego elementos nucleares de la Teoría del Apego (Bowlby, 1982); del SC (George y Solomon, 1996, 2008; Solomon y George, 1996) y de la Conexión (Bell, 2012; Bell y Richard, 2000).

Nuestros resultados generales iniciales apuntan a que los niños de estas edades (entre siete y nueve años), poseen ya una comprensión del SC de la madre. Es decir, poseen una representación de que la madre tiene como un objetivo prioritario procurar protección y bienestar al hijo. La no consecución de este objetivo o los impedimentos que pudieran estar presentes, elicitarían emoción negativa en la madre (enfado o preocupación) cuando no puede alcanzar dicho objetivo. Por otra parte, los datos obtenidos parecen señalar que es típico de estas edades poseer una representación bidireccional, diádica, entre el SC y el Sistema de Apego, puesto que los niños señalan como causa de la emoción de malestar de la madre, el sentimiento de desprotección o la necesidad del hijo de ser atendido por ella. Dentro de esta representación, hallamos que los niños tienen en cuenta las emociones de la madre ligadas a la necesidad de compatibilizar diferentes objetivos o necesidades, entre las que se encuentra la protección del niño, en la línea de la Teoría del Cuidado (Solomon y George, 1996) y no tenido en cuenta por la Teoría del Apego.

Las respuestas de los niños que representan el mayor nivel de complejidad en estas edades, se encuentran en la línea de Bell y Richard (2000) cuando señalan la responsabilidad y la empatía como las emociones que forman el núcleo del sistema representacional del Cuidado. Lo interesante es que los niños lo capten y formen parte de sus propias representaciones, ya que ambas emociones no siempre son de fácil observación directa. 
Así mismo, respecto a los componentes cognitivos y emocionales, la emoción es el primer aspecto que, de manera intuitiva y espontánea, conforma la Representación infantil del SC, de hecho, aparece ya, en el Nivel 1 de respuesta. En los siguientes niveles, aparecen, por una parte, el componente cognitivo (i.e. toma de conciencia, responsabilidad; aparición del dilema entre la responsabilidad y la situación ) y, de manera integrada la emoción de una forma cada vez más compleja e incluyendo emociones autoconscientes como la vergüenza que suponen la comparación con otros y con un estándar de "comportamiento deseable" (Fridja, 2008; Harris, 1989) y que, además, requieren un grado de abstracción mayor por parte del niño para captarlas e interpretarlas dentro del comportamiento del otro, en este caso, la madre. Estos argumentos se encontrarían en la línea de lo que Bowlby (1989) denomina Fase IV en la formación del Apego (Goal-corrected partnership), hacia los dos años, cuando comienza la formación del Sistema representacional del Apego en la que se conforma el Modelo Interno de Trabajo. Así mismo y, de nuevo, en relación al Sistema de Representación del Apego, Delius, Bovenschen y Spangler (2008) sostienen que, como parte de su representación del Apego, los niños incluyen los estados mentales (creencias, emociones, deseos, ...) de su figura de Apego. Nosotros encontramos estos contenidos también en el sistema de Representación de Cuidado que los niños tienen sobre la figura de apego.

Por otra parte, las explicaciones que ofrecen los niños, con sus diferentes grados de elaboración se encuentran en la línea de que, al igual que el conocimiento y la comprensión de otros fenómenos del mundo físico y social, no se trata de copias de la realidad (menos, cuando el sistema de representación es, por definición abstracto y no observable) sino que son construcciones activas de los niños (Main et al., 1985). Éstas, posiblemente, dependan no sólo de su desarrollo cognitivo sino también de sus experiencias afectivas personales en el ámbito de las relaciones de apego.

En relación con esto, desde una perspectiva evolutiva (Delval, 2000) y de las nociones sociales (Kalish y Lawson, 2008) podría señalarse que no todos los niños de entre siete y nueve años, tienen la misma representación del SC, sino que varían en cuanto a su cualidad y grado de complejidad, aunque mayoritariamente los niños de estas edades se encuentran en los niveles 2 y 3 . Desde estos planteamientos, quizá las representaciones del Nivel 1 sean más frecuentes en edades de niños anteriores y las propias del nivel 3 se consolidaran en edades posteriores. Esto nos ofrece nuevos e interesantes ámbitos de estudio.

Además, resulta también interesante el hecho de que cuando a los niños se les argumentaba que no era posible la solución propuesta para que la madre desplegara el SC, pudiera resultar un dinamizador de respuestas de un nivel más complejo sólo en aquellos niños que se encuentran en el nivel 3. Podría hipotetizarse que, ante un argumento que podría actuar como la "contrasugerencia" (Piaget, 1926) utilizada en la exploración de la representación infantil de conocimiento del mundo físico, sólo aquellos niños que poseen un sistema de representación más avanzado, son capaces de mantener dicha representación.

Desde una perspectiva aplicada, nuestro conocimiento de que los niños poseen ya en estas edades una representación del SC, que dicha representación es diádica y que contiene elementos de comprensión de la emoción y la cognición de la madre, resulta relevante. Desde la Teoría del Apego, se señala que la Representación del Apego (el Modelo Interno de Trabajo), una vez estructurada, gestiona de manera automática la percepción de los miembros de la díada, situándose fuera de la consciencia (Bowlby, 1973). Por ello, tienden a mantenerse estables, aunque hay posibilidad de modificación, lo que resulta especialmente importante para los niños cuyas experiencias de apego no han resultado saludables. Experiencias afectivas positivas con la misma figura de apego o con otras durante la infancia pueden facilitar un nuevo sistema de representación del apego saludable. Sin embargo, Main (1991) apunta que estas representaciones (y sus consecuencias) pueden modificarse únicamente mediante procesos de análisis y reinterpretación de las experiencias afectivas con su figura de apego. Para ello, son necesarias las competencias metacognitivas de la adolescencia. Por tanto, desde la Teoría de Apego, las representaciones del sistema de apego del niño pueden modificarse mediante experiencias que "compensen y sustituyan" un modelo no saludable. Sin embargo, este enfoque no contempla la posibilidad de la toma de conciencia por parte del niño de 
elementos del SC que, en determinadas condiciones, pudiera amortiguar la vivencia del niño de un comportamiento materno que pudiera ser vivido como no protector. Ello sería especialmente útil en el caso de díadas en las que su característica principal es la asincronía afectiva, el desencuentro entre las necesidades del niño y la respuesta de la madre (que se produce a destiempo o en una dirección equivocada) por razones de falta de competencia parental.

Entendiendo los sistemas de apego y de cuidado como complementarios, las madres pueden beneficiarse de conocer las representaciones y atribuciones que realiza su hijo sobre su comportamiento y los motivos/causas que lo determinan.

Por otra parte, el estudio de la comprensión infantil del SC, nos conducirá también a una mejor comprensión de cómo se construye las relaciones apego. Desde estos presupuestos, puede resultar una herramienta útil en la detección de riesgo o alteraciones en las relaciones de apego, a la vez, que una estrategia interesante en la detección e intervención en apegos no saludables.

Nuestro estudio tiene un carácter exploratorio y novedoso que debe de irse completando con investigaciones que profundicen en otros elementos $y$, desde una perspectiva evolutiva, en la comprensión infantil de este fenómeno crucial para el desarrollo, en edades anteriores y posteriores.

\section{Referencias}

Ainsworth, M. D. S. (1985). Patterns of Infant-Mother Attachments: Antecedents and Effects on Development. Bulletin of The New York Academy of Medicine, 61, 771-791

Bell, D. C. (2010). The Dynamics of Conection; How Evolution and Biology Create Caregiving and Attachment. New York, NY: Lexington Books.

Bell D. C. (2012). Next Steps in Attachment Theory. Journal of Family Theory \& Review, 4(4), 275-281. https://doi.org/10.1111/j.1756-2589.2012.00135.x

Bell, D. C. y Richard, A. J. (2000). Caregiving: The Forgotten Element in Attachment. Psychological Inquiry, 11(2), 69-83. https://doi.org/10.1207/S15327965PLI1102_01

Bowlby, J. (1969/1982). Attachment and loss, Vol. I, Attachment ( $2^{\mathrm{a}}$ ed.). New York, NY: Basic Books.

Bowlby, J. (1973). Attachment and loss, Vol. 2, Separation: Anxiety and Anger (Trad. Cast. La separación afectiva, Buenos Aires, Paidós, 1976). Londres, UK: Hogarth Press

Clarke-Stewart, K. A., Goossens, F. A., Allhusen, V. D. (2001). Measuring Infant-Mother Attachment: Is the Strange Situation Enough? Social Development, 10 (2), 143-168. https://doi.org/10.1111/14679507.00156

Crittenden, P. M. (1998). Dangerous Behavior and Dangerous Contexts: A 35-year Perspective on Research on the Developmental Effects of Child Physical Abuse. En P. K. Trickett y C. J. Schellenbach (Ed), Violence Against Children in the Family and the Community (pp. 11-38). Washington, DC: American Psychological Association

Delius, A., Bovenschen, I. y Spangler, G. (2008). The Inner Working Model as a "theory of attachment": Development during the Preschool Years. 
Attachment \& Human Development, 10(4), 395414. https://doi.org/10.1080/14616730802461425

Delval, J. (2000). Sobre la naturaleza de los fenómenos sociales. En K. Korta y F. García Madruga (Comps). Palabras. Victor Sánchez Zavala in memoriam [Words. Victor Sánchez Zavala in memoriam] (pp. 95-122). Bilbao, España: Servicio editorial de la UPV-EHU.

Delval, J. (2007). Aspectos de la construcción del conocimiento sobre la sociedad [Aspects of the construction of knowledge about society]. Revista de Investigación en Psicología, 10(1), 9-48.

Frijda, N. H. (2008). The Psychologists' Point of View. En Lewis, M, Haviland-Jones, J. y Feldman, L. Handbook of Emotions (pp. 68-87). Nueva York: Ed. Guilford.

George, C. y Solomon, J. (1996). Respresentational Models of Relationships: Links between Caregiving and Attachment. Infant Mental Health Journal, 17(3) 198-216. https://doi.org/10.1002/(SICI)10970355(199623)17:3\%3C198::AIDIMHJ2\%3E3.0.CO;2-L

George, C. y Solomon, J. (2008). The Caregiving Sistem. A Behavioral Sistems Approach to Parenting ( $2^{\mathrm{a}}$ ed.). En J. Cassidy y P. R. Shaver (Eds.), Handbook of Attachment: Theory, Research and Clinical Application (pp. 833-856). Nueva York, NY: Guilford.

Harris, P. L. (1992). Los niños y las emociones [Childens and emotions]. Madrid, España: Alianza Psicología Minor

Kalish, W. C. y Lawson, C. A. (2008). Development of Social Representations: Early Appretiation on Roles and Deontic Relations. Child Development, 79(3), 577-593.

Main, M. (1991). Metacognitive Knowledge, Metacognitive Monitoring, and Singular (coherent) vs. Multiple (incoherent) Models of Attachment:
Some Findings and some Directions for Future Research. En P. Marris, J. Stevenson-Hinde y C. Parkes (Eds.), Attachment Across the Life Cycle (pp. 127-159). Nueva York, NY: Routledge.

Main, M., Kaplan, K. y Cassidy, J. (1985). Security in Infancy, Childhood and Adulthood: A Move to the Level of Respresentation. En I. Bretherton y E. Waters (Eds.), Growing Points in attachment theory and research. Monographs of the Society for Research in Child Development, 50(209), 66-104. Chicago, IL: University of Chicago Press.

Mikulincer, M. y Shaver, P. R. (2005). Mental Representations of Attachment Security: Theoretical foundation for a Positive Social Psychology. En M. W. Baldwin (Ed.), Interpersonal cognition (pp. 233266). New York, NY: Guilford Press.

Piaget, J. (1926). El método clínico. Traducción de la introducción de La representación del mundo en el niño. En J. del Delval (Comp.) Lecturas de psicología del niño, vol I [Readings of child psychology]. Madrid, España: Alianza.

Solomon, J. y George, C. (1996). Defining the Caregiving System: Toward a Theory of Caregiving. Infant Mental Health Journal, 17(3), 183-197. https://doi.org/10.1002/(SICI)10970355(199623)17:3\%3C183::AIDIMHJ1\%3E3.0.CO;2-Q

Solomon, J., y George, C., (2000). Toward an Integrated Theory of Maternal Caregiving. En J. Osofsky y H.E. Fitzgerald (Eds.), WAIMH Handbook of Infant Mental Health, Vol. III: Parenting and Child Care (pp 323-368). Nueva York, NY: Wiley.

Walsh, T. M., Symons, D. K. y McGrath, P. J. (2004) Relations between Young Children's Responses to the Depiction of Separation and Pain Experiences. Attachment \& Human Development, 6, 53-71. 
